[0212-7199 (2004) 21: 7; pp 317-321] ANALES DE MEDICINA INTERNA Copyright $\odot 2004$ ARAN EDICIONES, S.L.

AN. MED. INTERNA (Madrid) Vol. 21, N. ${ }^{\circ}$, pp. $317-321,2004$

\title{
Mortalidad hospitalaria en un Servicio de Medicina Interna
}

\author{
C. SANCLEMENTE, M. BARCONS, M ${ }^{\mathrm{a}}$. A. MOLEIRO ${ }^{1}$, F. ALONSO, D. PAÑELLA, \\ R. CARRERA², R. TORIBIO, A. ANGLADA, J. VILARÓ
}

Servicio de Medicina Interna. ${ }^{1}$ Técnico de mortalidad. ${ }^{2}$ Unidad de Calidad. Hospital

General de Vic. Barcelona

\author{
HOSPITAL MORTALITY IN AN INTERNAL MEDICINE SERVICE
}

\section{RESUMEN}

El Servicio de Medicina Interna del Hospital General de Vic (Barcelona) forma parte del comité de mortalidad revisando y discutiendo los fallecimientos hospitalarios.

Fundamento y objetivo: Establecer las características de los fallecidos, causas de muerte y revisar si existen cambios en los últimos 6 años o problemas en relación con el éxitus, para evaluar y mejorar la asistencia de los pacientes ingresados.

Sujetos y métodos: Se realizó revisión de cada caso a través de un registro específico con:datos demográficos, diagnóstico y causa de muerte, problemas hospitalarios, datos de documentación,situación terminal/agónica al ingreso, si se realizó autopsia y datos de calidad de muerte.

Se analizan los éxitus secundarios a problemas hospitalarios (ESPHS).

Para el análisis estadístico se utilizaron las medidas de centralización y dispersión estándar.

Resultados: En los 6 años revisados hubieron 819 éxitus (5,1\%), la edad media global fue de $79 \pm 1,8$ años.el $52,5 \%$ eran varones y el $47,4 \%$ mujeres, el 22,8\% fallecieron en menos de 48 horas de ingreso.las causas más frecuentes de muerte fueron: el accidente vascular cerebral (24\%),la enfermedad pulmonar obstructiva crónica $(14,4 \%)$,la pneumonia (9,6\%).el número de autopsias fue muy reducido $(4,8 \%)$ los esphs se mantuvieron estables durante los 6 años, con una tasa del $0,5 \%$ siendo la infección nosocomial el problema más importante.

Conclusiones: El porcentaje total de éxitus fue del 5,1\%, superior a los estandards aconsejados. Las causas de mortalidad coinciden con otras series.

La tasa de ESPHs se ajustó a los objetivos recomendados.

El número de autopsias fue muy reducido.

La correcta cumplimentación y revisión de la historia clínica es imprescindible para detectar déficits en la asistencia de los pacientes ingresados.

PALABRAS CLAVE: Mortalidad hospitalaria. Eventos adversos. Autopsia. Calidad asistencial.

\section{ABSTRACT}

The Internal Medicine service of the Hospital General de Vic (Barcelona) takes part in the mortality committee by revising and discussing in-hospital mortality.

Background: To establish the characteristics of the deceased, death causes and to revise possible changes in the last six-years time or problems related to the exitus, to evaluate and improve hospitalized patients' assistance.

Methodology: Every case was revised following a specific register: demographical data, diagnosis and death cause, hospital death, documentation data, terminal or agonic situation when hospitalized, autopsies and death quality data.

Exitus due to hospital problems were analyzed and classified in different groups.

The statistical analysis was performed with measures of central tendency and of standard deviation.

Results: During the revised six years, there were 819 exitus (5.1\%). Global average death age was $79 \pm 1.8$ years: $52.5 \%$ were men and $47.4 \%$ were women; $22.8 \%$ died in less than forty-eight hours after hospitalization. The most frequent death causes were cerebrovascular accident $(24 \%)$, chronic obstructive pulmonary disease (14.4\%) and pneumonia (9.6\%). There were a small number of autopsies (4.8\%). Ratio of exitus due to hospital problems was stable during the six years $(0.5 \%)$, in which nosocomial infection was the severest problem.

Conclusions: The total percentage of exitus was $5.1 \%$, higher than the common standards. Mortality causes coincide with other series. Ratio of exitus due to hospital problems was according to recommended objectives. The number of autopsies was very small. A correct completing and revision of the clinical recording is indispensable to spot a shortage in the hospitalized patients' assistance.

KEY WORDS: In-hospital mortality. Adverse events. Autopsy. Assistance quality.

Sanclemente C, Barcons M, Moleiro MA, Alonso F, Pañella D, Carrera R, Toribio R, Anglada A, Vilaró J. Mortalidad hospitalaria en un Servicio de Medicina Interna. An Med Interna (Madrid) 2004; 21: 317-321.

Trabajo aceptado: 25 de febrero de 2004

Correspondencia: Carmen Sanclemente. Servicio de Medicina Interna. Hospital General de Vic . C/ Francesc Pla "El Vigatà", s/n. 08500 Vic (Barcelona). e-mail:csanclemente@hgv.es 


\section{INTRODUCCIÓN}

La mortalidad hospitalaria ha sido clasicamente uno de los indicadores de calidad asistencial más frecuentemente utilizado, el análisis de mortalidad hospitalaria puede utilizarse como un valioso instrumento para la planificación y gestión de los hospitales.

Es conocido que la cuantificación de las defunciones hospitalarias puede considerarse como una medida de efectividad de la intervención hospitalaria (1).

El análisis de la mortalidad en la literatura se inicia en 1976 cuando Rutstein D, y cols. Publicó un listado de afecciones en las que había evidencia científica, que era posible evitar la enfermedad, incapacidad y/o muerte, con los medios sanitarios disponibles ya sea a través del tratamiento o la prevención (2).

En la década de los 80 la Health Care Financing Administration incorporó el análisis del porcentaje de mortalidad hospitalaria como comparación cualitativa de los hospitales americanos. Desde entonces han sido muchos los estudios publicados en este sentido, coincidiendo en la necesidad de ajustar la mortalidad a otros parámetros $(3,4)$.

Las dificultades en la utilización de ratios de mortalidad global y ajustada, entorpece el poder realizar un análisis cualitativo de comparación entre hospitales. La necesidad de revisar historias clínicas para detectar deficiencias en la asistencia y la introducción del análisis de los efectos adversos revitalizan el concepto de muerte evitable y algunas de las estrategias que Rutstein D y cols. Ya formuló.

Diversos estudios analizan los efectos adversos debidos a la intervención sanitaria, que ocasionan algun tipo de discapacidad ya sea reversible o permanente $(3,5)$.

Desde 1985 el Servicio de Medicina Interna del Hospital General de Vic (Barcelona) forma parte del comité de mortalidad, dentro del programa de calidad asistencial del centro. Este comité está formado por médicos y enfermeras que discuten mensualmente todos los casos de mortalidad del hospital.

Objetivo: Establecer las características clínicas de los pacientes fallecidos, así como las causas de muerte en un servicio de Medicina Interna.

Revisar si existen cambios a lo largo de los últimos 6 años o problemas en relación con el éxitus con el fin de que su análisis permita mejorar la asistencia de los pacientes ingresados

En este trabajo queremos mostrar nuestra metódica de trabajo y los resultados obtenidos al revisar los éxitus desde 1997 al 2002.

\section{MATERIAL Y MÉTODO}

El Hospital General de Vic es un hospital comarcal situado en la provincia de Barcelona y que dispone de un total de 216 camas. El servicio de Medicina Interna genera aproximadamente 2628 altas /año en el centro.

El análisis de los datos de mortalidad son fruto de la revisión sistemática de cada caso, a través de un registro específico donde consta: datos demográ-ficos, diagnóstico y causa de la muerte, problemas hospitalarios, datos de documentación, situación terminal/agónica al ingreso, si se realizó necropsia así como datos acerca de calidad de muerte.

Se revisan todos los pacientes fallecidos en el servicio de
Medicina Interna desde enero de 1997 a diciembre de 2002.

Se analizan los éxitus secundarios a problemas hospitalarios (ESPHs) que se agrupan en: a) infección nosocomial; según la definición del Center Diseases Control (CDC); b) técnica diagnóstica/terapéutica; cuando la causa de muerte está directamente relacionada con la ejecución de una decisión diagnóstica o terapéutica; c) omisión de tratamiento/profilaxis; no proporcionar o demorar un tratamiento o profilaxis de demostrada eficacia, ya sea por error diagnóstico o desconocimiento; d) incidente nocivo; hechos inesperados que pueden suceder en un hospital y que pueden conducir a la muerte del paciente como por ejemplo las aspiraciones o caidas; y e) problema organizativo/estructural; cuando la muerte se puede atribuir directamente a un déficit organizativo o de estructura, por ejemplo un desfibrilador que no funcione.

Se recogen datos de calidad de muerte como: definición de muerte previsible, tratramiento no paliativo y sin beneficio previsible (fármacos, catéteres y drenajes, medidas quirúrgicas, y pruebas complementarias), tratamiento paliativo y si éste es eficaz, indicación de no reanimación cardiopulmonar (no RCP), información del desenlace al paciente y a los familiares, si el paciente se encuentra ingresado en la unidad de cuidados intensivos (UCI) en el momento del fallecimiento y si se ha intentado trasladar a una unidad de hospitalización, si el paciente se encontraba en habitación individual, si había presencia de acompañantes en el momento de la muerte (familiares y/o sanitarios).

Para el análisis estadístico se utilizaron las medidas de centralización y dispersión estándar. Se utilizó la Chi cuadrada con la corrección de Fisher para la comparación de las variable cualitativas.

\section{RESULTADOS}

Desde 1997 a diciembre de 2002 se han producido 819 éxitus representando un $5,1 \%$ de las altas hospitalarias. Durante el año 2000 y 2001 no se revisaron 24 y 14 casos respectivamente siguiendo unos criterios de inclusión de aquellos que era necesario revisar (Tabla I).

El análisis de los fallecidos muestra una edad media de 79. $1 \pm 1$. 8 con un predominio de varones $(52,5 \%$ vs $47,4 \%)$. La distribución anual por sexo y edad se muestra en (Figs. 1 y 2).

La estancia media fué de 8,62 \pm 0,40 días.

La muerte se produjo en menos de $48 \mathrm{~h}$ del ingreso en 187 pacientes $(22,8 \%)$, sin diferencias estadísticamente significativas en cuanto a edad, sexo o causas de mortalidad, respecto al global de los fallecimientos.

Las causas más frecuentes de mortalidad global fueron el accidente vascular cerebral $(24,4 \%)$, la enfermedad pulmonar obstructiva crónica $(14,4 \%)$, la neumonía $(9,6 \%)$, la insuficiencia cardiaca $(6,9 \%)$, la neoplasia $(5,2 \%)$ y la cirrosis hepática (3,7\%) (Fig. 3).

El número de necrópsias fue muy reducido comparado con el número de éxitus en los que hubiera estado indicada (Tabla II).

La correlación clínico-patológica global fue del 72,5\% y su distribución anual se muestra en la (Tabla III).

Los ESPHs se mantuvieron estables durante los 6 años, con una tasa global del $0,5 \%$ sin diferencias estadísticamente significativas (Fig. 4). La infección nosocomial fué el problema más importante (Tabla IV). 


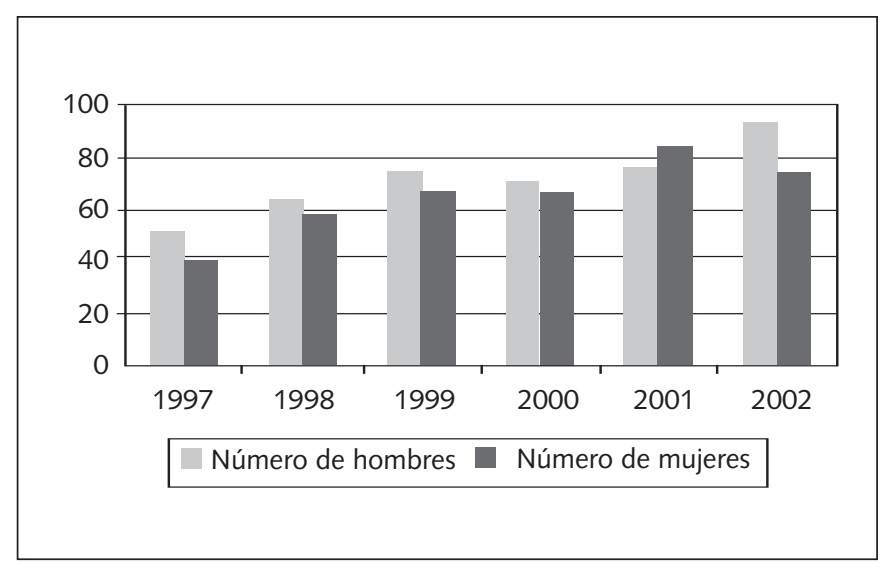

Fig. 1. Distribución anual del sexo.



Fig. 2. Distribución anual de la edad.



Fig. 3. Causas más frecuentes de mortalidad.

La información recogida sobre la calidad de la muerte de los pacientes en la historia clínica ha ido mejorando desde el año 1997 al 2002 pero no tenemos resultados de todas las variables ya que en los primeros años revisados no están bien reflejadas en la historia clínica del paciente (Tabla V).

En el momento del ingreso se catalogaron de enfermos terminales 225 pacientes $(28,8 \%)$.

En los 6 años analizados no se produjo ningún caso de muerte evitable definida ésta según los criterios de Rutstein (2).
TABLA I

CRITERIOS DE INCLUSIÓN DE AQUELLOS ÉXITUS QUE ES NECESARIO REVISAR

Edad $<65$ años

Reingresos en un periodo menor de 60 días

Los intervenidos quirúrigicamente

Los que tenían diagnóstico de sintomatologia*

Los éxitus producidos en menos de $48 \mathrm{~h}$ del ingreso

Con criterios de mortalidad evitable

Si se habia hecho necropsia

Ingreso previo en la $\mathrm{UCI}$

UCl: unidad de cuidados intensivos. ${ }^{*}$ Basado exclusivamente en síntomas

TABLA II

NÚMERO DE NECROPSIAS Y EXITUS CON INDICACIÓN DE NECROPSIA

\begin{tabular}{lcccccc}
\hline & 1997 & 1998 & 1999 & 2000 & 2001 & 2002 \\
\hline Necropsias & 8 & 3 & 9 & 10 & 4 & 6 \\
$\begin{array}{l}\text { Indicación } \\
\text { de necropsia }\end{array}$ & 26 & 27 & 61 & 26 & 35 & 56 \\
\hline
\end{tabular}

\section{DISCUSIÓN}

Los grandes avances tecnológicos de los que se dispone actualmente, no han proporcionado una disminución de la morbimortalidad. Es indiscutible que la aplicación de sistemas de evaluación en los centros hospitalarios, pueden ayudar a detectar problemas y a mejorar la calidad asistencial (6).

Existen pocos estudios en la literatura revisada sobre mortalidad hospitalaria en servicios de Medicina Interna y estos reflejan que la variabilidad en la mortalidad puede ser importante, la edad media de nuestros pacientes fue superior si la comparamos con otras series hospitalarias pero la distribución del sexo fue similar $(1,3,4)$. De esto se extrae que existe una gran dificultad en crear una tasa de mortalidad universalmente válida (7).

En nuestro servicio de Medicina Interna, durante el periodo estudiado se produjo un $5,1 \%$ de fallecimientos, porcentaje 
TABLA IV

\begin{tabular}{|c|c|c|c|c|c|c|c|}
\hline \multicolumn{8}{|c|}{ DISTRIBUCIÓN DE LOS ESPHS } \\
\hline & 1997 & 1998 & 1999 & 2000 & 2001 & 2002 & total \\
\hline $\begin{array}{l}\text { Omisión o demora de } \\
\text { tratamiento o } \\
\text { profilaxis }\end{array}$ & $5(41,6 \%)$ & $2(13,3 \%)$ & $1(6,2 \%)$ & 0 & 0 & $2(11,1 \%)$ & $10(11,7 \%)$ \\
\hline Infección nosocomial & $2(16,6 \%)$ & $5(33,3 \%)$ & $8(50 \%)$ & $12(92,3 \%)$ & $5(45,4 \%)$ & $9(50 \%)$ & $41(48,2 \%)$ \\
\hline Broncoaspiración & $2(16,6 \%)$ & $5(33,3 \%)$ & $4(25 \%)$ & 0 & $3(27,2 \%)$ & $4(22,2 \%)$ & $18(21,1 \%)$ \\
\hline Otros & $3(25 \%)$ & $3(20 \%)$ & $3(18,7 \%)$ & $1(7,6 \%)$ & $3(27,2 \%)$ & $3(16,6 \%)$ & $16(18,8 \%)$ \\
\hline
\end{tabular}

TABLA $\vee$

CALIDAD DE MUERTE N(\%)

\begin{tabular}{ll}
\hline Definición de muerte previsible & $713(86,3 \%)$ \\
Tratamiento paliativo & $650(91,5 \%)$ \\
Tratamiento paliativo eficaz & $643(98,8 \%)$ \\
Indicación de no RCP & $651(90,4 \%)$ \\
Información a familiares & $662(92,8 \%)$ \\
\hline
\end{tabular}

RCP:reanimación cardiopulmonar

ligeramente superior al recomendado por el Instituto Nacional de Salud (4\%). Las causas de mortalidad hospitalaria se producen por enfermedades muy prevalentes en nuestro medio, no modificandose en los 6 años estudiados, siendo similares al resto de las series $(1,3,4)$.

La práctica clínica de autopsias es muy antigua, hay datos que indican su práctica en el Antiguo Egipto y en la Edad media. Los beneficios que aporta la autopsia al desarrollo científico de la medicina son innegables. En los últimos 50 años el porcentaje de autopsias ha descendido de una manera alarmante $(8,9)$, este problema también lo hemos detectado en nuestro centro y confirmado al revisar nuestra serie, con un porcentaje muy reducido de autopsias realizadas $4,8 \%$, si lo comparamos con el número en que el grupo de mortalidad consideró que estaba indicada, una vez evaluada la historia clínica. La causa es multifactorial en donde están implicados los clínicos, familiares del paciente y organización hospitalaria entre otros. Creemos que es necesario que se produzcan cambios en la actitud en todos aquellos que están directa o indirectamente implicados en la autopsia, ya que ésta es un procedimiento objetivo para detectar errores diagnósticos y es importante su utilidad como método de control de calidad (10, $11)$.

El análisis de la correlación clínico-patológica pone de manifiesto la existencia de enfermedades detectadas en la autopsia, que no fueron detectadas en vida del paciente; en nuestra serie encontramos una correlación clínico-patológica global del $72,5 \%$. Consideramos muy importantes las reuniones conjuntas con el servicio de Anatomia patológica para llevar a cabo el análisis de estos datos y así mejorar los resulta$\operatorname{dos}(12)$.

Al revisar la literatura hemos encontrado que se utiliza diferente terminología para expresar una misma idea (ESPH), así podemos encontrar: complicaciones, yatrogenia, efectos adversos, patología nosocomial o problemas hospitalarios. Además los criterios de inclusión de cada estudio son diferentes incluso utilizando palabras iguales. Pocos estudios incluyen el concepto de omisión o demora del tratamiento o profilaxis como en nuestro análisis.

La tasa global de ESPH en nuestro servicio fue del 0,5\% dato que se ajusta a los objetivos recomendados.

Respecto al análisis de la distribución de los tipos de ESPHs detectados están en relación a los publicados en la literatura, destacando en primer lugar la infección

nosocomial a expensas principalmente de la pneumonia, coincidiendo con otras series (13).

Estamos convencidos de que el sistema de detección y análisis de ESPHs es objetivo y un buen sistema de trabajo en calidad asistencial que además sirve para instaurar un sistema de vigilancia de los problemas asistenciales que inciden directamente sobre la muerte de los pacientes. Serian precisos más estudios para poder corroborar la utilidad de los ESPHs como indicador de calidad y poder generalizar su utilización.

Destacamos la necesidad de seguir revisando historias clínicas, de forma similar a la que realiza nuestro grupo, como método de fuente de datos para la detección de posibles deficiencias en la calidad asistencial del sistema sanitario.

La revisión de estas historias clínicas es el único sistema para explicar diferencias de mortalidad en una misma patología en centros hospitalarios diferentes, detectando como única explicación deficiencias en la calidad asistencial. La correcta cumplimentación por parte del personal sanitario y la posterior revisión de la historia clínica es imprescindible para detectar déficits en la calidad asistencial. En nuestro trabajo objetivamos un escaso registro de las condiciones humanas de la muerte (habitación individual, presencia de familiares y/o sanitarios, entre otros)y creemos que se ha de mejorar para conocer su realidad. El acuerdo de calidad de muerte se realiza conjuntamente con la familia, pocas veces con el paciente, aunque pueden influir múltiples razones, esto pone de manifiesto la necesidad de una ética preventiva que proporcione elementos a los pacientes crónicos para conocer su situación y perspectiva para ayudarlo a adecuar sus expectativas a la realidad y participar activamente en la toma de decisiones.

En resumen consideramos que los datos obtenidos en nuestra revisión pueden ayudarnos sin duda a mejorar la calidad asistencial de nuestros pacientes y poder disminuir o evitar eventos que desencadenan o aumentan la mortalidad hospitalaria de los mismos. 


\section{Bibliografía}

1. García Ortega C, Almenara Barrios J, García ortega JJ. Ratios específicas de mortalidad en el Hospital de Algeciras durante 1995-1996. Rev Esp Salud Pública. 1997; 71 (3): 305-15.

2. Rutsein DD, Berenberg W, Child CG, Fishman, AP, Perrin EB. Measuring the quality of medical care. N Engl J Med 1976; 11: 582-8.

3. De Escalante Yanguela B, Oncins Torres R, Lacasa Marzo J, Candel Calderon M, Sanpedro Feliu JA. Estudio de mortalidad hospitalaria en el Servicio de Medicina Interna de un hospital comarcal. An Med Interna (Madrid) 1994; 11: 381-4.

4. Del Río Fernández MC, Fernández Renedo A, de Frutos Arribas JF, Acebes Rey JM, Martín Escudero JC, Martínez Barrero F. Mortalidad en un Servicio de Medicina Interna de un hospital de tercer nivel: veinte años de experiencia. An Med Interna (Madrid) 1998; 15: 197-201.

5. "Avoidable" causes of death in Sweden 1974-85. Assur Health Care 1992; 4 (4): 319-28.

6. Moty C, Michel P. Organizational and operational work of death analysis committes: review of experiences. Presse Med 2001; 30 (6): 259-63.
7. Dubois RW, Brook RH, Rogers WH. Adjusted Hospital Death Rates: A potential screen for quality of medical care. Am J Public Health 1987; 77: 1162-6

8. Pelletier LL Jr, Klutzow F, Lancaster H. The autopsy: its role in the evaluation of patient care. J Gen Intern Med 1989; 4 (4): 300-3

9. Ferguson RP, Burkhardt L, Hennawi G, Puthumana L. Consecutive autopsies on an internal medicine service. South Med J 2004; 97 (4): 335-7.

10. Belzer A, Rivkind Al. It always pays to make a diagnosis: To autopsy or not to autopsy? Crit Care Med 1998; 26: 1299-300.

11. Abrahan E. Autopsies in the UCI: We still need them. Crit Care Med 1999; 27 (2): 235-6.

12. Bombí J. A. Número de autopsias clínicas y correlación clínico-patológica. Rev Esp Patol 2004; 37 (1): 5-12.

13. Rodríguez-Rumayor G, Fernández C, Delgado A, Carrasco M, Andradas E, De Juan S, et al. Relación de la infección nosocomial con la mortalidad hospitalaria. Estudio multicéntrico. Med Clin (Barc) 1993; 100: 9-13. 